\title{
Different roles of lateral anterior temporal lobe and inferior parietal lobule in coding function and manipulation tool knowledge: Evidence from an rTMS study
}

\author{
Ryo Ishibashi a,b, Matthew A. Lambon Ralph ${ }^{\mathrm{b}}$, Satoru Saito ${ }^{\mathrm{a}, \mathrm{b}}$, Gorana Pobric ${ }^{\mathrm{b}, *}$ \\ a Department of Cognitive Psychology in Education, Kyoto University, Japan \\ ${ }^{\mathrm{b}}$ Neuroscience and Aphasia Research Unit, University of Manchester, UK
}

\section{A R T I C L E I N F O}

\section{Article history:}

Received 18 August 2010

Received in revised form

15 December 2010

Accepted 4 January 2011

Available online 8 January 2011

\section{Keywords:}

Semantic memory

Anterior temporal lobe

Tool function

Tool manipulation

rTMS

\begin{abstract}
A B S T R A C T
A remarkable cognitive ability in humans is the competency to use a wide variety of different tools. Two cortical regions, the anterior temporal lobes (ATL) and left inferior parietal lobule (IPL), have been proposed to make differential contributions to two kinds of knowledge about tools - function vs. manipulation. We used repetitive transcranial magnetic stimulation (rTMS) and two semantic decision tasks to assess the role of these regions in healthy participants. Participants made semantic decisions about the function (what for) or manipulation (how) of tools used in daily life. The stimulation of ATL resulted in longer responses for the "function" judgments, whilst stimulation of IPL yielded longer responses for the "manipulation" judgments. In line with the neuropsychological literature, these results are discussed within hub-and-spoke framework of semantic memory.
\end{abstract}

(c) 2011 Elsevier Ltd. All rights reserved.

\section{Introduction}

The ability to use a wide variety of tools is one of the characteristic features of humans. However, how this cognitive ability is implemented neurally is still unclear. It has been suggested that a ventral and a dorsal pathway are involved in the interpretation of "what" and "how" for manmade objects (Fang \& He, 2005; Goodale \& Milner, 1992). The two visual systems carry out different transformations on incoming visual information (Goodale \& Milner, 1992). According to this view, the dorsal stream, which projects to posterior parietal cortex, utilizes moment-to-moment information about the disposition of objects within egocentric frames of reference and mediates goal directed acts, while the ventral route is specialised for processing of action knowledge. This model has been refined by Rizzolatti and Matelli (2003) who proposed a system that codes visual information for action organization. The anatomical data and a reconsideration of previous functional and clinical data, implicates that the dorsal stream and its recipient parietal areas form two distinct functional systems: the dorso-dorsal stream (d-d stream) and the ventro-dorsal stream ( $\mathrm{v}-\mathrm{d}$ stream). The $\mathrm{d}$ - $\mathrm{d}$ stream plays a major role in the control of actions "on line". Its damage leads

\footnotetext{
* Corresponding author at: Neuroscience and Aphasia Research Unit, School of Psychological Sciences, University of Manchester, 3rd floor Zochonis Building, Brunswick Street, Manchester M13 9PL, UK. Tel.: +44 01612751967.

E-mail address: gorana.pobric@manchester.ac.uk (G. Pobric).
}

to optic ataxia. The d-d stream is specialized for on-line control of grasping. The $\mathrm{v}-\mathrm{d}$ stream is formed by area MT (main $\mathrm{v}$ - $\mathrm{d}$ extrastriate visual node) and by the visual areas of the inferior parietal lobule. As the d-d stream, v-d stream is responsible for action organization. It, however, also plays a crucial role in space perception and action recognition. Both $\mathrm{d}-\mathrm{d}$ stream and $\mathrm{v}$-d stream are distinct from the ventral stream dedicated to object recognition (Buxbaum \& Kalénine, 2010).

In the "hub-and-spoke" framework of semantic cognition (Lambon Ralph, Sage, Jones, \& Mayberry, 2010; Patterson, Nestor, \& Rogers, 2007), semantic representations are formed and activated through the joint action of different types of information. Like the classical (e.g. Wernicke-Meynert) and contemporary (distributed only) approaches to semantic memory the "spokes" represent the information that arises in each of our sensory, motor and verbal association cortices. These are entry and exit points for both comprehension and expression of semantic knowledge whether in the verbal or nonverbal domain. If these were only directly connected to each other, they might be able to generate some concepts through the amalgamation of features, but they would not be able to generalize across concepts that have similar semantic significance but not necessarily similar specific attributes (Lambon Ralph et al., 2010; Patterson et al., 2007). In the hub-and-spoke model, this challenge is met by the addition of an extra, intermediating representational layer (McClelland \& Rogers, 2003). This allows the formation of amodal multidimensional representations that, through the translation of information between each modal- 
ity, code the higher-order statistical structure that is present in our experience. It is "amodal" not in the sense of forming symbolic representations but because these representations are not linked to any particular modality. Instead, the representations are modalityinvariant (Lambon Ralph et al., 2010). As well as being able to code the partial similarity structure in specific domains, the modalityinvariant representations add greater flexibility in order to deal with concepts that do not follow these surface similarities; and the system provides a mechanism for generalisation to new or changing exemplars. In short, the addition of an amodal hub, allows the system to code for semantic family resemblances present in and across each modality-specific spoke (Lambon Ralph et al., 2010). The ATL is a potentially suitable substrate for the neuroanatomical implementation of a modality-invariant hub because of two key features: (a) it is not associated with any single motor, sensory or verbal input/output but (b) it is widely connected to other temporal, parietal and frontal regions (Gloor, 1997) allowing different streams of modality-specific information to converge on this point.

In this study, we investigated the roles of two distinct neural regions (IPL and ATL) within the dorsal and ventral streams in the context of the "hub-and-spoke" model of semantic cognition. According to the "hub-and-spoke" model (Lambon Ralph et al., 2010; Pobric, Jefferies, \& Lambon Ralph, 2010a; Rogers \& McClelland, 2004), these two regions support conceptual information about manmade objects in different ways. The ATL hub is involved in the translation and deeper encoding of pan-modal information sources, resulting in representations that are modalityinvariant and thus involved in conceptualisation for all types of knowledge. In contrast in the IPL spoke, modality-specific information only contributes to the subset of concepts that are experienced in that modality. Under the classical distributed views and the contemporary hub-and-spoke framework, multiple various regions contribute different sources of information towards conceptualisation. In this context we are assuming that a sub-region of the parietal lobe contribute important praxis-related information. This does not mean that the parietal region is only implicated in this function but rather than one of its roles is the coding of this kind of information.

While it is generally accepted that impaired tool use in apraxic patients may be the result of damage to one of several distinct cognitive processes, an influential model of apraxia states that when a learned motor skill is performed, a knowledge representation of the motor skill is retrieved from the parietal lobes rather than constructed anew, and then it is projected forward to the motor association areas and ultimately to the motor cortex (Heilman \& Rothi, 1993). If an object is to be manipulated correctly (not only grasped), then dorsal-ventral stream (including left IPL) is engaged (Barde, Buxbaum, \& Moll, 2007). The researchers showed that apraxic patients are impaired in grasp-to-use, but not grasp-to-move, suggesting that these two action systems are separable (Barde et al., 2007; Buxbaum, Sirigu, Schwartz, \& Klatzky, 2003). Consistent with this patient data, neuroimaging evidence has shown that grasp-to-use (Buxbaum, Kyle, Tang, \& Detre, 2006) and familiar tool use knowledge (Vingerhoets, 2008) shows activations in the inferior parietal cortex. In addition to being associated with grasp-to-use, the neuroimaging studies have shown greater activation in the inferior parietal cortex for manipulation than function judgments (Boronat et al., 2005), suggesting that impaired grasp-to-use in apraxia is closely related to impaired manipulation knowledge. Furthermore, it has been argued that while both function and manipulation knowledge require semantic tool knowledge, manipulation knowledge may also rely on regions that mediate skilled tool use (Buxbaum \& Saffran, 2002). Therefore, we hypothesised that IPL is a good area to test manipulation knowledge (e.g. grasp-to-use). To date, at least four research areas have provided converging evidence in favour of this working hypothesis.

\subsection{Neuropsychological evidence}

The neuropsychological literature provides a series of reports on patients with diminished ability to recognize and/or to use tools. Damage to left inferior parietal lobule (IPL) has often been associated with ideational apraxia - an impairment in using familiar objects that cannot be attributed to physical dysfunction of the limbs (Rothi \& Heilman, 1997). Investigations to date reveal that patients with ideational apraxia have degraded knowledge about how to move their body parts to interact with tools, while often being able to identify the function of those tools correctly, indicating that such patients maintain knowledge of the function of common objects (Buxbaum \& Saffran, 2002; Buxbaum, Veramonti, \& Schwartz, 2000; Rosci, Chiesa, Laiacona, \& Capitani, 2003). The patients reported in these studies usually had large infarction of the left parietal cortex, around the IPL area. Therefore, it is considered that their behavioural deficit stems from the damage to this cortical area.

Different kinds of object-use deficits can be observed in patients with a neurological disorder associated with general conceptual degradation. Semantic dementia (SD) patients have bilateral atrophy and hypometabolism, which is maximal in the inferior and lateral aspects of the ATLs. Moreover, the extent of this atrophy correlates with the severity of the semantic impairment (Mummery et al., 2000; Nestor, Fryer, \& Hodges, 2006). Studies of SD patients suggest that the patients suffer from impaired knowledge of how to use common tools as well as their purpose (Bozeat, Lambon Ralph, Patterson, Garrard, \& Hodges, 2000; Bozeat, Lambon Ralph, Patterson, \& Hodges, 2002; Hodges, Bozeat, Lambon Ralph, Patterson, \& Spatt, 2000). The degree of the patients' objectuse deficit has been found to correlate with their lower scores of general semantic recognition and comprehension. In addition, exploration of the performance in patients with varying degrees of SD has revealed significant item-based correlations between semantic knowledge and object use accuracy, probed with the same set of manmade objects (Bozeat et al., 2002; Hodges et al., 2000). These studies support the notion that the ATL is associated with every type of semantic knowledge including praxis of objects.

While various studies have found a very tight yoking of semantic knowledge and tool use, most studies have found that poor performance on the semantic tasks tends to precede the decline in the ability to use objects. In longitudinal studies (e.g., Coccia, Bartolini, Luzzi, Provinciali, \& Lambon Ralph, 2004) this yields two parallel declining curves, with semantic performance always somewhat lower than tool use. As a consequence, in the very early phase of ATL damage, some SD patients with mild impairment show relatively preserved performance in using familiar objects (Coccia et al., 2004; Silveri \& Ciccarelli, 2009), despite mild, albeit observable impairments on harder semantic tests. In summary, the neuropsychological literature indicates that lesions to the IPL generate a specific deficit to tool manipulation knowledge while the ATL generates a general, pan-modal effect which, in the context of tool use, impacts on both function and praxis in a parallel fashion.

\subsection{Neuroimaging evidence}

Several neuroimaging investigations of healthy participants have reported dissociable neural activations associated with either function knowledge or knowledge of manipulation of tools. Kellenbach, Brett, and Patterson (2003) conducted a positron emission tomography (PET) study to investigate the neural activation associated with making judgments about tools' function or manipulation. They found increased activation in left inferior parietal cortex when participants were asked to make binary decisions about the actions associated with familiar objects (e.g. "Does using the object involve a back-and-forth action?") relative to when they 
were making judgments about the function of the same objects (e.g. "Is the object used to attach or hold objects together?"). Similar activation in the left parietal area was also reported by Boronat et al. (2005) in a functional magnetic resonance imaging study (fMRI). They presented pairs of tool pictures and asked the participants to judge whether or not the tools had the same function (e.g., matches and lighter), or if they could be manipulated in a same way (e.g., piano and computer keyboard). The comparison of the neural activations for these two tasks indicated significantly higher activation in the left inferior parietal area (extending from angular gyrus to inferior parietal sulcus) during the manipulation-relative to the function-related judgments (Boronat et al., 2005). Using very similar tasks, Canessa et al. (2008) found contrasting activations in parietal and inferior temporal cortices during the manipulation and function judgments, respectively. The authors reported significantly higher activation for manipulation than function task in the left IPL, premotor cortex and inferior parietal sulcus. In contrast, the inferior temporal lobe was activated significantly more in the function judgments (Canessa et al., 2008). These neuroimaging findings are consistent with the different neuropsychological symptoms associated with the damage in these structures (see above), implying that there is a relative division of labour across the left inferior parietal and inferior temporal areas, with respect to the knowledge and use of familiar tools.

\subsection{TMS evidence}

Through the use of repetitive transcranial magnetic stimulation (rTMS) in normal participants, Pobric et al. (2010a) demonstrated two contrasting effects of stimulation to the lateral ATL vs. IPL. Stimulation of the ATL led to a generalised slowing of semantic processing across all types of concepts (living, manipulable objects and non-manipulable man-made items). This is in keeping with the category-general deficits observed in the context of the ATLfocussed atrophy underlying semantic dementia (Lambon Ralph, Lowe, \& Rogers, 2007). In contrast, stimulation of the IPL generated a category-specific pattern reflective of the praxis information underpinned by this neural region. These results are consistent with the category-specific pattern observed in stroke patients with lesions in this same region (Buxbaum \& Saffran, 2002). This category-general effect generated by TMS of the ATL was explained by the hub-and-spoke model, which assumes that the ATL combines various perceptual and motor representations distributed throughout the brain (Patterson et al., 2007).

\subsection{Behavioural evidence}

Several behavioural experiments also support the idea that parietal and temporal areas have different cognitive roles in tool recognition (Almeida, Mahon, \& Caramazza, 2010; Almeida, Mahon, Nakayama, \& Caramazza, 2008). Almeida et al. (2010) reported that a category-specific priming effect on naming manmade objects was evoked by presentation of tools under continuous flash suppression (CFS). CFS disrupts visual processing only in the ventral pathway while keeping the dorsal pathway unaffected (Tsuchiya \& Koch, 2005). Consequently, the category-specific priming effect under CFS, observed by Almeida et al. (2010) implies that the "how" (dorsal stream) object information continues even when participants are not aware of the visual presentation.

In conclusion, all of four research areas, neuropsychological, neuroimaging, TMS and cognitive psychology, indicate that the ventral (temporal cortex) and dorsal (parietal cortex) areas are differentially associated with the cognitive processing of tool knowledge. While ATL serves as a circuit for the conceptual representation of tools, IPL contributes specifically to praxis information. However, there are some key inherent limitations with the current literature. First, with respect to the patient studies, the precision of the anatomical inference is always limited by the generally large lesions found in neurological patients and also by the impact of post-damage strategies and effects of recovery. Likewise, whilst providing good spatial resolution, functional neuroimaging studies do not reveal the necessity of each region to normal function (Price \& Friston, 2002). These two important sources of neuroscience data are complemented by rTMS studies because both the location and the extent of the stimulation effect are under experimental control.

\subsection{Aim of the current study}

The aim of this study was to investigate the interaction of the hub (ATL) and a spoke (IPL) during a semantic judgment task. We studied the roles of two distinct neural regions in processing explicit knowledge of common tools utilizing rTMS. We used this method to temporally interfere with the neural activity over left lateral ATL or left IPL in healthy participants while they were making judgments about tools in terms of their function (for what purpose they are used) or manipulation (how they are used). With this virtual-lesion method, we intended to delineate between two alternative predictions. One prediction is that the stimulation of the ATL will affect both the function and manipulation knowledge, while the effect TMS on IPL will be observed for the manipulation knowledge only. This would be in line with the general semantic deficits observed in SD patients and specific deficits observed in apraxia patients. An alternative prediction is that TMS over ATL will affect only decisions relating to function knowledge in line with the neuroimaging literature and patients with mild SD. With milder levels of damage (as in patients with mild SD, or transient TMS stimulation), the interaction between hub and spokes may be more important, as the spoke-based information may be able to support or influence the partially degraded hub representations (e.g., Coccia et al., 2004). Either result would indicate different roles for ATL and IPL in processing knowledge about everyday object use.

\section{Methods}

\subsection{Participants}

Thirteen participants took part in the present experiment ( 6 females; mean age $=27.08$ years, $S D=7.02$ ). All were native English speakers and eleven of them were right-handed. All participants had no history of neurological or mental disorders and gave informed consent on their participation in the experiment and having magnetic stimulation. The experiment was conducted under the review and approval of the local research ethics board.

\subsection{Task}

The tasks were derived from clinical investigations of tool use knowledge in semantic dementia patients (Bozeat et al., 2002; Corbett, Jefferies, \& Lambon Ralph, 2009; Hodges et al., 2000). To use the test in a TMS study, we aimed to make the task difficult enough for neurologically intact participants such that rTMS might induce both RT and accuracy effects on performance. We developed three cognitive tasks for this investigation - function matching, manipulation matching, and visual-pattern matching. All of them required participants to select a target word (function and manipulation tasks) or pattern (visual matching task). In the functionand manipulation-matching tasks, a probe word (e.g., scissors) was presented at the top of the screen and other words appeared underneath. One of them was a tool that had the same function as the probe (e.g., guillotine) while another one was a tool which was manipulated in the same way as the probe (e.g., staple). The last word had no semantic relationship to the probe (e.g., whisk; see Fig. 1 and Appendix B). Participants were asked to choose the target that had the same function (in function matching condition) or the same way of manipulation (in manipulation matching condition) as the probe. The visual pattern matching task is a control task. It provides a non-semantic control condition that can be compared with other semantic tasks in order to correctly interpret the TMS effects. The task was designed to be structurally identical to the semantic tasks but without requiring any semantic processing. In the visual-pattern matching task, a scrambled probe picture was presented and participants were asked to indicate which of three patterns presented underneath was an inverted image of the probe. Our previous TMS study (Pobric et al., 2010a; Pobric Jefferies, \& Lambon Ralph, 2010b) adopted a similar task and found no interference effect of TMS on this non-semantic task performance. 
<Semantic matching tasks>

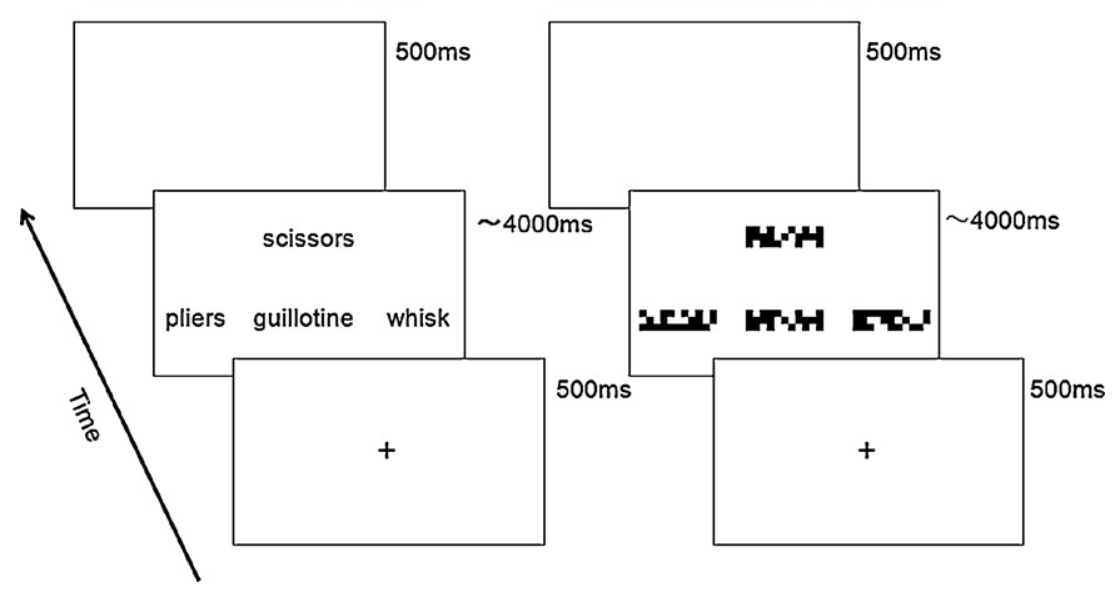

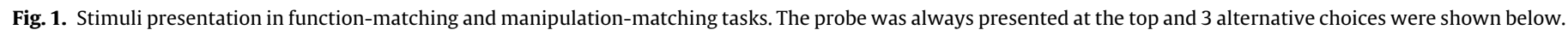

\subsection{Stimuli}

One hundred and sixty-one common tool names were used as stimuli in the function and manipulation matching tasks. To choose appropriate combinations of stimuli, we conducted a preliminary investigation in which we tested 176 trials with different probes, function targets and manipulation targets. Participants were asked to press a key for the target, which had either the same function (F) or manipulation (M) feature as the probe. They were tested on both tasks and eighty trials were chosen by eliminating the items with the lowest accuracy. Reaction times (RTs) were generally longer in manipulation than in function matching task $[F(1,79)=44.4$ $p<.01]$. Unpaired $t$-tests confirmed that the mean decision times for the separate sets were not significantly different in either function matching $[t(78)=.11, p>.90]$ or manipulation matching $[t(78)=1.51, p>.10]$. The probe objects were used in both pre- and post-TMS sessions in the real experiment but with different semantically unrelated foils (see Fig. 1). We developed a visual-pattern matching task (V) as a control task. For this task, scrambled pictures of the probe words in the two semantic matching tasks were utilized. The stimuli were produced by dividing the images of words into $5 \times 5$ tiles which were subsequently scrambled. In the task, the probe picture was presented at the top of the screen with other scrambled pictures underneath. In order to match this non-semantic task for global difficulty, the target was an inverted image of the probe item. The other two foils were different scrambled visual patterns of the probe. There were 240 trials in total in a single TMS session (each participant had three TMS sessions - IPL, ATL, OCC separated by at least three week period). There were 80 trials per condition/block (functional matching, manipulation matching and visual pattern matching). These 80 trials were divided in pre-TMS blocks ( 40 trials) and post-TMS blocks ( 40 trials). The blocks were presented pseudo-randomly: participants saw three blocks of 40 trials for each condition ( $F$, $\mathrm{M}, \mathrm{V}$ ) before TMS, and another 3 blocks $\times 40$ trials of $(\mathrm{F}, \mathrm{M}, \mathrm{V})$ after receiving $10 \mathrm{~min}$ of $1 \mathrm{~Hz}$ rTMS. Therefore each participant saw all 240 trials in a single session, but the blocks were randomised. At the start of each block/condition, there was a clear instruction about the task: function, manipulation or visual matching. Participants also had practice trials that followed the same blocked design before the experimental session. Previous studies have indicated that the neurophysiological effect of $1 \mathrm{~Hz}$ rTMS applied on motor cortex generally exceeds duration of the stimulation train (Muellbacher, Ziemann, Boroojerdi, \& Hallett, 2000; Robertson, Théoret, \& PascualLeone, 2003). However, when $1 \mathrm{~Hz}$ rTMS is applied to a different brain area (e.g. somatosensory cortex), behavioural effects are much shorter (Knecht et al., 2002). We were expecting the suppression to last for $6-8 \mathrm{~min}$. The 4 blocks of testing were $14 \mathrm{~min}$ long (avg.). The TMS condition lasted on average for $7 \mathrm{~min}$.

\subsection{TMS}

Transcranial magnetic stimulation was applied using a MagStim Rapid2 (Magstim Co., Whitland, UK) stimulator with 2 external boosters (maximum output was approximately $2.2 \mathrm{~T}$ ). A $70-\mathrm{mm}$ figure-of-eight coil was placed on a predetermined position on the participant's scalp. To establish the position of the coil, the participants' T1-weighted MRI scans were co-registered with their scalp using MRIreg (www.mricro.com/mrireg.html) and an Ascension minibird magnetic tracking system (www.ascension-tech.com). Coordinates for ATL and IPL were determined for each participant prior to the experiment. The location of ATL was $10 \mathrm{~mm}$ posterior to the anterior tip of the left temporal lobe along the middle temporal gyrus. The average coordinates for ATL corresponded to $(-51,9,-21)$ in the Montreal Neurological Institute (MNI) standard space. The location of IPL was obtained from a previous fMRI study that tested the knowledge of tool manipulation with healthy participants (Kellenbach et al., 2003). The MNI coordinates in left inferior parietal area that showed maximum activation in judging manipulation of tools in the study $(-38,-44,48)$ were converted to each individual subject's native space using SPM5 (http://www.fil.ion.ucl.ac.uk/spm/). In order to interpret the TMS effects, it is important to show that similar effect would not arise by stimulating just any other brain area. This is why we employ the control site. In this case we wanted to be sure that the control site in not part of the functional semantic network. The occipital site is fairly distant from other two areas (IPL and ATL) and has already been used in other TMS studies on semantics (Fuggeta, Pobric, Rizzo, Lavidor, \& Walsh, 2009; Knecht et al., 2002; Pobric, Jefferies, \& Lambon Ralph, 2007; Pobric et al., 2010a, Pobric, Lambon Ralph, \& Jefferies, 2009). Occipital pole was determined based on physical landmarks. The coil was positioned at $20 \mathrm{~mm}$ above and $10 \mathrm{~mm}$ left to the inion along the surface of the participants' scalp in this condition. Stimulation on this area has already been proved to cause no advert effect on semantic processing in our previous TMS study (Pobric et al., 2010a, 2010b).

Motor thresholds were determined for every participant by acquiring the minimum intensity of stimulation on the optimal position to induce contraction of the relaxed contralateral abductor pollicis brevis muscle. Their motor thresholds ranged from 40 to $64 \%$ of the maximum stimulator output. Stimulation was delivered at $120 \%$ of motor threshold but kept at $67 \%$ of the device's maximum output if it exceeded this criterion (This occurred in $16.7 \%$ of the sessions). Average stimulation intensity was $58.8 \%$. Participants received 10 -min of TMS stimulation $(1 \mathrm{~Hz}$ for $600 \mathrm{~s}$ ). This TMS protocol has been shown to produce behavioural effects that last for several minutes after stimulation (Eisenegger, Treyer, Fehr, \& Knoch, 2008; Hilgetag, Theoret, \& Pascual-Leone, 2001; Kosslyn et al., 1999). The stimulation was carried out with particular care to minimize any unpleasant sensations. Following our previously adopted procedure (Pobric et al., 2007, 2010b), the coil orientation was modified for each participant to minimise facial muscle twitching and any discomfort. All participants were chosen from a dedicated subject pool in the University of Manchester and were pre-screened on their ability to tolerate this kind of stimulation.

\subsection{Procedure}

A fixation point was presented for $500 \mathrm{~ms}$ at the beginning of each trial to signal its start. Subsequently, the stimuli were presented and remained on the screen until the participants made a response or until 4000 ms elapsed. A $500 \mathrm{~ms}$ blank screen (the inter-trial interval) followed. Participants were asked to press the key that corresponded to the target $(1,2$, or 3$)$ with the index, middle, or ring finger of their dominant hand and were instructed to make the response as quickly and accurately as possible. Each experimental session included all three tasks (function, manipulation and visual-pattern matching). All tasks were conducted both before and after TMS ( 6 blocks in total, three pre and three post TMS), thus any non-specific effects of TMS, such as noise, tactile sensation and muscle twitching, did not overlap with task performance. The order of tasks was pseudo-randomized but kept same between pre and post TMS session. The successive stimulation of each neural location was achieved on three separate days, with site being counterbalanced across days.

\section{Results}

\subsection{Response time analysis}

Trials where a participant failed to respond in $4000 \mathrm{~ms}$ or gave an incorrect answer were removed from the data prior to the following analysis ( mean failure rate $=4.3 \%$; mean error rate $=12.1 \%$ ). Fig. 2 shows the mean RTs in all 18 experimental conditions. 


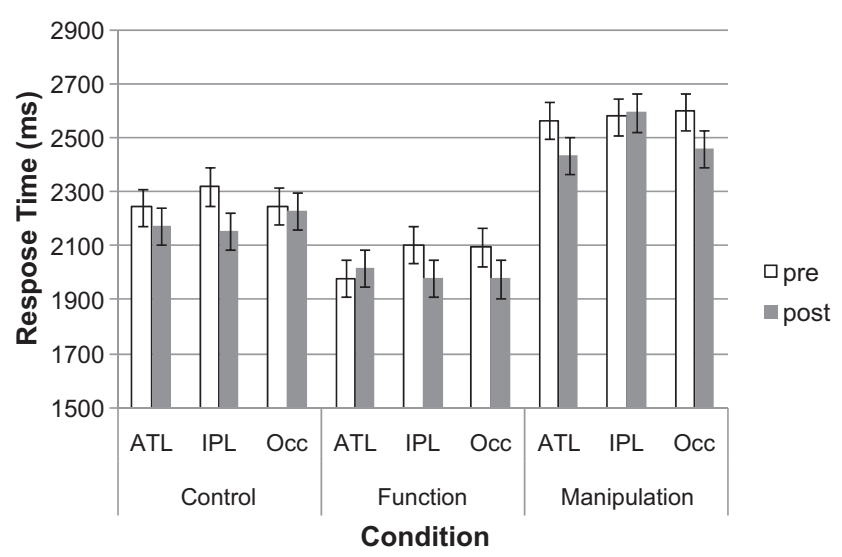

Fig. 2. Response times for control (visual pattern), function, and manipulation matching tasks. Error bars indicate confidence interval, adjusted for within subject comparisons (Loftus \& Masson, 1994).

A three-way analysis of variance (ANOVA) with task (function, manipulation, control), site (ATL, IPL, Occ) and TMS (pre-TMS, post-TMS) as within-subject factors was performed. There were main effects of task $[F(2,18)=27.19, p<.01]$ and TMS $[F(1,9)=16.65$, $p<.05]$. Subsidiary $t$-test comparisons showed that RTs in manipulation judgment task was significantly longer than that in visual pattern matching task $[t(24)=4.44, p<.05]$ Also the difference in RTs of visual pattern and function matching tasks was also significant $[t(24)=2.88, p<.05]$. Importantly, there was a significant three-way interaction $[F(4,48)=3.45, p<.05]$ between task, site and TMS.

As reported in previous rTMS experiments (cf. Holland \& Lambon Ralph, 2010; Pobric et al., 2007), there was a substantial practice and general alerting effect of TMS, which confounded the more specific TMS effect (the pre-TMS baseline sessions always preceded the post-TMS sessions). In fact, in seven of nine conditions (task by site), the RTs were faster in the second (post-TMS) than that in the first (pre-TMS) session. Two exceptions, however, were observed: longer RTs in the second (post-TMS) session for the function matching condition after ATL-stimulation and longer RTs for the manipulation matching condition after IPL-stimulation (see Fig. 2).

\subsection{Error analyses}

The error rate was examined in a repeated measures ANOVA with tasks (function, manipulation and visual-pattern matching), site (ATL, IPL, Occ) and TMS (pre-TMS, post-TMS) as factors. There were no significant main effects or interactions [ $p$ 's $>.1$ ].

To partial out the confounding effects from the TMS effect, we utilized the occipital (control site) stimulation data as a baseline. We have demonstrated previously that TMS in this region has no effect on semantic judgments (Pobric et al., 2010b) and so it serves as a suitable control site and the data arising from this condition can be used to partial out the practice/alerting effect. RTs in this condition were subtracted from corresponding RTs in ATLand IPL-stimulation conditions to acquire the deviation data from baseline (Holland \& Lambon Ralph, 2010). Fig. 3 shows the resultant, corrected-RT data. A three-way ANOVA revealed a significant three-way interaction $[F(2,24)=3.95, p<.05]$. Planned comparisons revealed a significant slowing effect of TMS in the function matching task after ATL-stimulation $[F(1,72)=4.44, p<.05]$ and for the manipulation matching decisions after IPL-stimulation $[F(1,72)=4.35, p<.05]$. No other differences were significant.

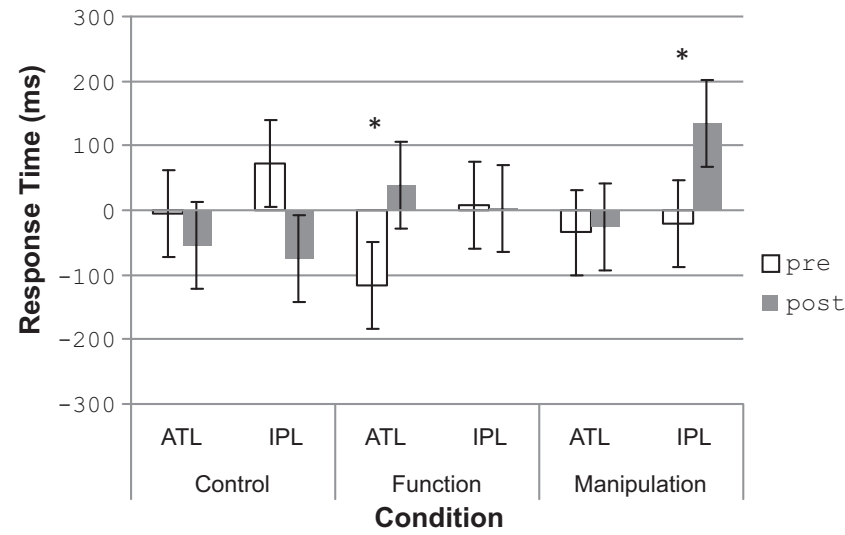

Fig. 3. Deviations of RTs in ATL and IPL condition with corresponding RTs in OCC condition as baseline. Error bars indicate standard errors, adjusted for within subject comparisons (Loftus \& Masson, 1994).

\section{Discussion}

In the present study, we found an rTMS-induced reaction time slowing in two cognitive tasks probing different kinds of knowledge about tools. Magnetic stimulation of the left IPL caused longer RTs in a manipulation-judgment task, while stimulation of the left ATL slowed performance on the function-matching task. This was explored with exactly the same stimuli for both tasks and within the same participants, excluding the possibility of attributing the results to different item features or inter-individual variations. In addition, neither IPL nor ATL-stimulation affected performance in visual-pattern matching. The results clearly demonstrate an interactive partnership between modality-specific sources of information (spokes) and modality-invariant representations required for the formation of coherent concepts (the hub) (see Lambon Ralph et al., 2010). The results are in accordance with the hub-and-spoke model of semantic cognition (McClelland \& Rogers, 2003; Patterson et al., 2007; Pobric et al., 2010a, 2010b). We discuss in the following sections the roles of IPL and ATL in relation to use of familiar manmade objects.

\subsection{Role of IPL in object manipulation}

The stimulation of left IPL significantly increased RTs in the manipulation matching task but did not have an effect on the other judgements. This result is in accordance with the reports of tooluse deficits after cortical damage in left parietal areas (Buxbaum et al., 2000; Rosci et al., 2003). The present results support the view that IPL plays a role in retrieving a particular movement for using familiar tools and explains why lesions of this area cause loss of tool-use ability as shown by apraxic patients. IPL's activation is reported in studies of various cognitive properties, including visual attention (Hahn, Ross, \& Stein, 2006), saccadic eye movements (Pierrot-Deseilligny, Rivaud, Gaymard, Müri, \& Vermersch, $1995)$ and visual working memory (Courtney, Ungerleider, Keil, \& Haxby, 1996). IPL is also implicated in translating visual information into motor programs to achieve imitations (Molenberghs, Cunnington, \& Mattingley, 2009; Mühlau et al., 2005). In addition, IPL is regarded as a part of the mirror neuron system which activates both for perception and execution of particular movements (Buccino et al., 2004). Given these perceptual and motor properties associated with the IPL, this area can be considered as an interface between perceptual and motor information.

However, whether or not this area stores representations of movements for actual tool use is still a matter of debate. In a recent study, Goldenberg and Spatt (2009) suggested that the left parietal 
cortex processes spatial relationship between objects and bodyparts rather than storing praxis knowledge. Their lesion analysis with 34 left-hemisphere damaged patients has shown that the lesion volume in left inferior parietal cortex correlates more with the score of novel-tool use than with common-tool use. In addition, Goldenberg, Hermsdörfer, Glindemann, Rorden, and Karnath (2007) indicated that IPL damage is not associated with tool-use gestures. Thus this study concluded that the left inferior parietal area does not store memory for manipulating familiar objects. Though these data seem to provide counter evidence of our results, a few points would be worth mentioning. Firstly, the lesion study recruited patients with various areas of lesions in the left hemisphere, including frontal and temporal lobes. It is quite difficult, therefore, to attribute patients' performance to a single site. In fact, each of lesions in middle frontal, supramarginal, precentral, and inferior parietal areas significantly correlated with the scores of novel and common tool-use tasks (Goldenberg \& Spatt, 2009). Although the tool-use scores in this cross-sectional study was not explained by the entire lesion size of each participants' brain, as covariance between two individual lesion sites can substantially differ from each area's covariance with total lesion size, it might be possible that the effect of one lesion site indirectly influence correlation of another lesion site, without having high covariance with total lesion size. Thus, the results could be reflecting the contribution of another lesion site whose lesion size was positive correlated with that of IPL or combined effect of multiple infarctions including IPL. Secondly, often function, following neurological damage, can be recovered by other intact cortical structures in the brain (Ward, 2005). The repetitive TMS method, used in current study, provides an important additional source of information over and above neuropsychological-only studies because the position of the "virtual lesion" area is under experimental control and the behavioural effects can be tested immediately after stimulation. Our data show impaired performance on tool manipulation decisions after stimulation of left IPL. Therefore, we can conclude that the area is causally involved in retrieving knowledge of tool manipulation. We should note, however, that the results do not exclude the possibility that this area also works for other cognitive processes in object use, such as grasping or the spatial relationship between tool and body-parts. In fact, apraxic patients with relatively spared IPL can competently learn novel tool-use actions when there are enough mechanical cues (affordance) (Barde et al., 2007), indicating that IPL may be responsible for acquisition of new object-related movements anchored in mechanical structure.

\subsection{Role of ATL in object recognition}

Stimulation of the left ATL increased response times for semantic judgments in the function-matching task, but not that for the manipulation-matching task. In keeping with the fact that rTMS produces a mild effect typically measured in terms of slowed RTs rather than by the increased error rates observed in patients, the effect of ATL-TMS in the function-judgment task is compatible with the findings from very mild SD patients (Coccia et al., 2004; Silveri \& Ciccarelli, 2009). At this very early stage, patients can demonstrate use for very familiar items but already exhibit mild impairment on semantic tasks - including the naming and comprehension of objects. Longitudinally, both tool use and semantic knowledge decline in parallel but with use always somewhat better than semantic performance (e.g. Coccia et al., 2004). This differential performance between object use and object knowledge, in mild SD, is mirrored in the results reported in the current study and in our previous rTMS work (Lambon Ralph, Pobric, \& Jefferies, 2009; Pobric et al., 2007, 2010b). In all these studies we stimulated the same ATL region using the same stimulation protocol. Previously, we found a clear effect on difficult semantic tasks such as synonym or association judgements, and a small but significant effect in simple picture naming - even of tools. In the current study we found an effect on tool function decisions without an effect on tool manipulation judgements.

Our results are also in accordance with the spatial relationship framework (Goldenberg, 2009). According to this proposal, the hand is a link in a chain leading from bodily actions to the external effect of the tool. An object can be manipulated in a consistent way with kinaesthetic restrictions of human body. As a result, there are some groups of tools which can be used in a similar way but have different functions (e.g. scissors, stapler and tongs). It is conceivable that, compared to the various functions that should be associated with tools (e.g. binding papers, cutting, pinching small things, respectively), body movements to use those tools are selected from relatively smaller number of options. This nature in hand actions could be the reason that makes performance of object use relatively resistant to transient stimulation of the ATL hub.

Furthermore, our ATL-results are in agreement with several neuropsychological studies reporting patients with temporal lobe lesions (Buxbaum \& Saffran, 2002; Magnie, Ferreira, Giusiano, \& Poncet, 1999; Negri, Lunardelli, Reverberi, Gigli, \& Rumiati, 2007; Sirigu, Duhamel, \& Poncet, 1991). In these studies, patients with lesions in bilateral ATL could not efficiently retrieve the function of common tools, while they showed a spared ability to use them. It has been proposed that ATL is the structure that combines different types of modality-specific information through its connections with diffuse modality-specific circuits (Binney, Embleton, Jefferies, Parker, \& Lambon Ralph, 2010; Patterson et al., 2007). Considering that the function of tools arises from various properties of those objects (i.e. shape of a basket, sound of a rattle, colour of correcting fluid, etc.), the current data are compatible with this hub interpretation of the role of ATL.

On the other hand, there are a small number of cross-sectional studies of SD that indicate that the ATL damage leads to observable object-use deficits, something we did not observe in our RT-data. In a case-series study reported by Bozeat et al. (2000) the patients with semantic dementia showed impairment for using tools to the extent that is commensurate with their semantic deficit. Another case-series SD study with exactly the same set of 20 manmade objects for all tasks reported middle-to-high positive correlations between scores of semantic and motor tasks (Hodges et al., 2000). It also showed that a tool could no longer be used properly when the conceptual memory of the item had been completely lost.

How can we reconcile this apparent disparity between tool use and semantic performance after TMS stimulation over the ATL and in the SD patient data? At least two possibilities arise. First, the key variable moderating SD performances is the severity of the disorder. In the study by Silveri and Ciccarelli (2009), the researchers investigated general semantic and object-use performances of five patients who had mild to moderate temporal-lobe damage and observed almost perfect object-use performances from three mildseverity patients with bilateral temporal-lobe damages, while two mid-severity patients showed substantial degradation in their tool use (Silveri \& Ciccarelli, 2009). This indicates that the atrophy of ATL at the very early stage of SD is not sufficient to evoke an observable tool-use deficit, but it does arise when the disorder has progressed to a more severe level (Bozeat et al., 2002; Coccia et al., 2004; Hodges et al., 2000). Since rTMS can have only a weak and transitive effect at the site of stimulation, we could assume that the results of ATL-TMS in manipulation judgment mirrored only the very mild cases of SD but not the severe cases.

Second, we should take into account that object use receives a boost from object affordances, independent of semantics. The two-stream theory of visual perception (Goodale \& Milner, 1992; Jeannerod, Arbib, Rizzolatti, \& Sakata, 1995) proposes that visual information of an object can be processed through both ventral 
and dorsal streams in the brain. According to this theory, calculation for physically interacting with objects is executed by dorsal part of the brain extending from occipital to posterior parietal areas, while processing for recognizing objects is achieved by the ventral stream, which extends from occipital to inferior temporal cortices. Buxbaum and Kalénine (2010) recently refined this theory in the light of latest neuropsychological and neuroimaging studies and proposed the two action systems (2AS) account, in which dorsal stream is divided into two distinguishable subcomponents; dorsodorsal and ventro-dorsal streams. In this framework, dorso-dorsal pathway, located in bilateral IPS and dorso-lateral fronto-parietal areas, is regarded as a system that provide online information processing to create motor responses to objects based on affordances given by them. In contrast, ventro-dorsal pathway, located at left superior temporal and parietal areas, is assumed to provide core features of skilled actions with objects. These two dorsal streams, that include inferior and superior parietal area, are likely to be involved in calculation of the potential object-hand interaction. The direct visual-motor activation provides a potential boost to the computation of object-specific use, independent of the ventral stream, including the ATL - and this would explain why object use is always somewhat better than object knowledge in SD patients and in the current rTMS study. Evidence in favour of this hypothesis was provided by Bozeat et al. (2002). They compared features of object use that were either reliably predicted by the visual form of the object (e.g., handles $\rightarrow$ hold) or elements that were not reliably predicted (e.g., holding the hammer at the far end of the shaft). They found that the severity of the semantic impairment in the SD patients was highly predictive of the latter, non-"afforded" elements but had no relationship with the "afforded" aspects of the object's use (for which the patients' performance was excellent, even in severely impaired patients). Though the stimulus words in current study did not provide any visual features of the real objects, their retrieved visual representation could have affected the performance on manipulation matching task through the dorsal streams.

\subsection{Conclusion}

This study extends past neuropsychological and neuroimaging studies of object-use by providing clear evidence for an interactive partnership between modality-specific sources of information and modality-invariant representations required for the formation of coherent concepts. Interference with the neural processing in the IPL affected manipulation judgments about objects, mirroring apraxic patients with parietal lobe damage. In contrast, rTMS over the ATL caused slowed performance in the judgments of tool function, mirroring performance of mild SD patients. These results and the existing evidence from patients with ATL and IPL damage are best explained by the hub-and-spoke framework of semantic memory (Patterson et al., 2007). Under this account concepts reflect the combination of two sources of information - modalityspecific knowledge coded in their respective association cortices and the action of the ATL, re-representational hub. Such a partnership explains effects of both ATL and IPL stimulation on semantic processing for praxic related concepts although the exact outcome is to some extent different.

\section{Acknowledgments}

This research was supported in part by Japan Society for the Promotion of Sciences Research Fellowship for Young Researchers (21-7135), by the Alumni Association of the Kyoto University Department of Education, and by an MRC programme grant to MLR (G0501632).

\section{Appendix A. Supplementary data}

Supplementary data associated with this article can be found, in the online version, at doi:10.1016/j.neuropsychologia.2011.01.004.

\section{References}

Almeida, J., Mahon, B. Z., \& Caramazza, A. (2010). The role of the dorsal visual processing stream in tool identification. Psychological Science, 21, 772-778.

Almeida, J., Mahon, B. Z., Nakayama, K., \& Caramazza, A. (2008). Unconscious processing dissociates along categorical lines. Proceedings of the National Academy of Sciences of the United States of America, 105, 15214-15218.

Barde, L. H. F., Buxbaum, L. J., \& Moll, A. (2007). Abnormal reliance on object structure in apraxics' learning of novel object-related actions. Journal of the International Neuropsychological Society, 13, 997-1008.

Binney, R. J., Embleton, K. V., Jefferies, E., Parker, G. J. M., \& Lambon Ralph, M. A. (2010) The inferolateral aspects of the anterior temporal lobe are crucial in semantic memory: Evidence from a novel direct comparison of distortion-corrected fMRI rTMS and semantic dementia. Cerebral Cortex, 20, 2728-2738.

Boronat, C. B., Buxbaum, L. J., Coslett, H. B., Tang, K., Saffran, E. M., Kimberg, D. Y., et al (2005). Distinctions between manipulation and function knowledge of objects: Evidence from functional magnetic resonance imaging. Cognitive Brain Research 23, 361-373.

Bozeat, S., Lambon Ralph, M. A., Patterson, K., Garrard, P., \& Hodges, J. R. (2000). Non-verbal semantic impairment in semantic dementia. Neuropsychologia, 38 , 1207-1215.

Bozeat, S., Lambon Ralph, M. A., Patterson, K., \& Hodges, J. R. (2002). When objects lose their meaning: What happens to their use? Cognitive Affective E Behavioral Neurosciences, 2, 236-251.

Buccino, G., Vogt, S., Ritzl, A., Fink, G. R., Zilles, K., Freund, H. J., et al. (2004). Neural circuits underlying imitation learning of hand actions: An event-related fMRI study. Neuron, 42, 323-334.

Buxbaum, L. J., \& Kalénine, S. (2010). Action knowledge, visuomotor activation, and embodiment in the two action systems. Annals of the New York Academy of Sciences, 1191, 201-218.

Buxbaum, L. J., Kyle, K. M., Tang, K., \& Detre, J. A. (2006). Neural substrates of knowledge of hand postures for object grasping and functional object use: Evidence from fMRI. Brain Research, 1117, 175-185.

Buxbaum, L. J., \& Saffran, E. M. (2002). Knowledge of object manipulation and object function: Dissociations in apraxic and nonapraxic subjects. Brain and Language 82, 179-199.

Buxbaum, L. J., Sirigu, A., Schwartz, M. F., \& Klatzky, R. (2003). Cognitive representations of hand posture in ideomotor apraxia. Neuropsychologia, 41, 1091-1113.

Buxbaum, L. J., Veramonti, T., \& Schwartz, M. F. (2000). Function and manipulation tool knowledge in apraxia: Knowing 'what for' but not 'how'. Neurocase, 6, 83-97.

Canessa, N., Borgo, F., Cappa, S. F., Perani, D., Falini, A., Buccino, G., et al. (2008). The different neural correlates of action and functional knowledge in semantic memory: An fMRI study. Cerebral Cortex, 18, 740-751.

Coccia, M., Bartolini, M., Luzzi, S., Provinciali, L., \& Lambon Ralph, M. A. (2004) Semantic memory is an amodal, dynamic system: Evidence from the interaction of naming and object use in semantic dementia. Cognitive Neuropsychology, $21,513-527$

Corbett, F., Jefferies, E., \& Lambon Ralph, M. A. (2009). Exploring multimodal semantic control impairments in semantic aphasia: Evidence from naturalistic object use. Neuropsychologia, 47, 2721-2731.

Courtney, S. M., Ungerleider, L. G., Keil, K., \& Haxby, J. V. (1996). Object and spatial visual working memory activate separate neural systems in human cortex Cerebral Cortex, 6, 39-49.

Eisenegger, C., Treyer, V., Fehr, E., \& Knoch, D. (2008). Time-course of "off-line" prefrontal rTMS effects - A PET study. Neuroimage, 42, 379-384.

Fang, F., \& He, S. (2005). Cortical responses to invisible objects in the human dorsal and ventral pathways. Nature Neuroscience, 8, 1380-1385.

Fuggeta, G., Pobric, G., Rizzo, S., Lavidor, M., \& Walsh, V. (2009). Uncovering the dissociation between natural and artifactual domains: ERPs/TMS study. Journa of Cognitive Neuroscience, 21, 403-412.

Gloor, P. (1997). The temporal lobe and limbic system. Oxford: Oxford University Press.

Goldenberg, G., Hermsdörfer, J., Glindemann, J., Rorden, R., \& Karnath, C. H. O. (2007) Pantomime of tool use depends on integrity of left inferior frontal cortex. Cerebral Cortex, 17, 2769-2776.

Goldenberg, G., \& Spatt, J. (2009). The neural basis of tool use. Brain, 132, 1645-1655

Goldenberg, G. (2009). Apraxia and the parietal lobes. Neuropsychologia, 47 $1449-1459$

Goodale, M. A., \& Milner, A. D. (1992). Separate visual pathways for perception and action. Trends in Neurosciences, 15, 20-25

Hahn, B., Ross, T. J., \& Stein, E. A. (2006). Neuroanatomical dissociation between bottom-up and top-down processes of visuospatial selective attention. Neuroimage, 32, 842-853.

Heilman, K. M., \& Rothi, L. J. G. (1993). Apraxia. In K. M. Heilman, \& E. Valenstein (Eds.) Clinical neuropsychology (pp. 141-163). New York: Oxford University Press.

Hilgetag, C. C., Theoret, H., \& Pascual-Leone, A. (2001). Enhanced visual spatial attention ipsilateral to rTMS-induced 'virtual lesions' of human parietal cortex. Nature Neuroscience, 4, 953-957. 
Hodges, J. R., Bozeat, S., Lambon Ralph, M. A., Patterson, K., \& Spatt, J. (2000). The role of conceptual knowledge in object use evidence from semantic dementia. Brain, 123, 1913-1925.

Holland, R., \& Lambon Ralph, M. A. (2010). The anterior temporal lobe semantic hub is a part of the language neural network: Selective disruption of irregular past tense verbs by rTMS. Cerebral Cortex, 20, 2771-2775.

Jeannerod, M., Arbib, M. A., Rizzolatti, G., \& Sakata, H. (1995). Grasping objects: The cortical mechanisms of visuomotor transformation. Trends in Neurosciences, 18 314-320.

Kellenbach, M. L., Brett, M., \& Patterson, K. (2003). Actions speak louder than functions: The importance of manipulability and action in tool representation. Journal of Cognitive Neuroscience, 15, 30-46.

Knecht, S., Flöel, A., Dräger, B., Breitenstein, C., Sommer, J., Henningsen, H., et al (2002). Degree of language lateralization determines susceptibility to unilateral brain lesions. Nature Neuroscience, 5, 695-699.

Kosslyn, S. M., Pascual-Leone, A., Felician, O., Camposano, S., Keenan, J. P., Thompson, W. L., et al. (1999). The role of area 17 in visual imagery: Convergent evidence from PET and rTMS. Science, 284, 167-170.

Lambon Ralph, M. A., Lowe, C., \& Rogers, T. T. (2007). Neural basis of category-specific semantic deficits for living things: Evidence from semantic dementia, HSVE and a neural network model. Brain, 130, 1127-1137.

Lambon Ralph, M. A., Pobric, G., \& Jefferies, E.(2009). Conceptual knowledge is underpinned by temporal pole bilaterally: Convergent evidence from rTMS. Cerebral Cortex, 19, 832-838.

Lambon Ralph, M. A., Sage, K., Jones, R. W., \& Mayberry, E. J. (2010). Coherent concepts are computed in the anterior temporal lobes. Proceedings of the National Academy of Sciences of the United States of America, 107, 2717-2722.

Loftus, G. R., \& Masson, M. E. J. (1994). Using confidence intervals in within-subject designs. Psychonomic Bulletin E Review, 1, 476-490.

McClelland, J. L., \& Rogers, T. T. (2003). The parallel distributed processing approach to semantic cognition. Nature Reviews Neuroscience, 4, 310-322.

Magnie, M. N., Ferreira, C. T., Giusiano, B., \& Poncet, M. (1999). Category specificity in object agnosia: Preservation of sensorimotor experiences related to objects. Neuropsychologia, 37, 67-74.

Molenberghs, P., Cunnington, R., \& Mattingley, J. B. (2009). Is the mirror neuron system involved in imitation? A short review and meta-analysis. Neuroscience and Biobehavioral Reviews, 33, 975-980.

Muellbacher, W., Ziemann, U., Boroojerdi, B., \& Hallett, M. (2000). Effects of lowfrequency transcranial magnetic stimulation on motor excitability and basic motor behavior. Clinical Neurophysiology, 111, 1002-1007.

Mühlau, M., Hermsdorfer, J., Goldenberg, G., Wohlschlager, A. M., Castrop, F., Stahl R., et al. (2005). Left inferior parietal dominance in gesture imitation: An fMR study. Neuropsychologia, 43, 1086-1098.

Mummery, C. J., Patterson, K., Price, C. J., Ashburner, J., Frackowiak, R. S., Hodges, J. R., et al. (2000). A voxel-based morphometry study of semantic dementia: Relationship between temporal lobe atrophy and semantic memory. Annals of Neurology, 47, 36-45.
Negri, G. A., Lunardelli, A., Reverberi, C., Gigli, G. L., \& Rumiati, R. I. (2007). Degraded semantic knowledge and accurate object use. Cortex, 43, 376-388.

Nestor, P. J., Fryer, T. D., \& Hodges, J. R. (2006). Declarative memory impairments in Alzheimer's disease and semantic dementia. NeuroImage, 30, 1010-1020.

Patterson, K., Nestor, P.J., \& Rogers, T. T. (2007). Where do you know what you know? The representation of semantic knowledge in the human brain. Nature Reviews Neuroscience, 8, 976-987.

Pierrot-Deseilligny, C., Rivaud, S., Gaymard, B., Müri, R., \& Vermersch, A. I. (1995). Cortical control of saccades. Annals of Neurology, 37, 557-567.

Pobric, G., Jefferies, E., \& Lambon Ralph, M. A. (2007). Anterior temporal lobes mediate semantic representation: Mimicking semantic dementia by using rTMS in normal participants. Proceedings of the National Academy of Sciences of the United States of America, 104, 20137-20141.

Pobric, G., Jefferies, E., \& Lambon Ralph, M. A. (2010a). Category-specific versus category-general semantic impairment induced by transcranial magnetic stimulation. Current Biology, 20, 964-968.

Pobric, G., Jefferies, E., \& Lambon Ralph, M. A. (2010b). Amodal semantic representations depend on both anterior temporal lobes: Evidence from repetitive transcranial magnetic stimulation. Neuropsychologia, 48, 13361342

Pobric, G., Lambon Ralph, M. A., \& Jefferies, E. (2009). The role of the anterior temporal lobes in the comprehension of concrete and abstract words. Cortex, 45 1104-1110.

Price, C. J., \& Friston, K. J. (2002). Degeneracy and cognitive anatomy. Trends in Cognitive Sciences, 6, 416-421.

Rizzolatti, G., \& Matelli, M. (2003). Two different streams form the dorsal visual system: Anatomy and functions. Experimental Brain Research, 153, 146-157.

Robertson, E. M., Théoret, H., \& Pascual-Leone, A. (2003). Studies in cognition: The problems solved and created by transcranial magnetic stimulation. Journal of cognitive neuroscience, $15,948-960$.

Rogers, T. T., \& McClelland, J. L. (2004). Semantic cognition: A parallel distributed processing approach. Cambridge: MIT Press.

Rosci, C., Chiesa, V., Laiacona, M., \& Capitani, E. (2003). Apraxia is not associated to a disproportionate naming impairment for manipulable objects. Brain and Cognition, 53, 412-415.

Rothi, L. J. G., \& Heilman, K. M. (1997). Apraxia: The neuropsychology of action. London: Psychology Press.

Silveri, M. C., \& Ciccarelli, N. (2009). Semantic memory in object use. Neuropsychologia, 47, 2634-2641.

Sirigu, A., Duhamel, J. R., \& Poncet, M. (1991). The role of sensorimotor experience in object recognition. A case of multimodal agnosia. Brain, 114, 2555-2573.

Tsuchiya, N., \& Koch, C. (2005). Continuous flash suppression reduces negative afterimages. Nature Neuroscience, $8,1096-1101$.

Vingerhoets, G. (2008). Knowing about tools: Neural correlates of tool familiarity and experience. NeuroImage, 40,1380-1391.

Ward, N. S. (2005). Neural plasticity and recovery of function. Progress in Brain Research, 150, 527-535. 